08.02 - Transplantation

\title{
26134
}

\section{Clamshell vs bilateral anterolateral thoracotomy for double lung transplantation: impact on vital capacity}

Cystic fibrosis, Spirometry, Idiopathic pulmonary fibrosis

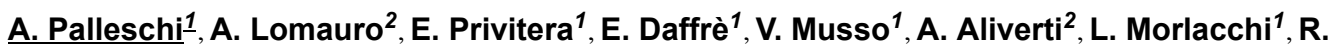 \\ Carrinola $^{1}$, V. Rossetti ${ }^{1}$, M. Nosostti ${ }^{3}$ \\ ${ }^{1}$ Fondazione IRCCS Ca' Granda - Ospedale Maggiore Policlinico of Milan - Milano (MI) (Italy), \\ ${ }^{2}$ Politecnico di Milano - Milano (Italy), ${ }^{3}$ Fondazione IRCCS Ca' Granda - Ospedale Maggiore \\ Policlinico of Milan - Milano (Italy)
}

\begin{abstract}
Objective: The bilateral anterolateral thoracotomy without sternal splitting is considered the preferable approach for bilateral lung transplantation, mainly for the reported lower rate of sternal complications and better respiratory function in the early postoperative period. We evaluate the influence of the incision on vital capacity within one-year follow up.
\end{abstract}

Methods: 133 double lung transplantations were done since January 2013 (85 cystic fibrosis, 36 interstitial lung diseases, 12 COPD). We retrospectively analysed spirometry performed before (pre), one month (post1), 6 months (post 6) and one year (post 12) after the transplantation. Forced vital capacity (FVC) was considered both as percentage predicted and as absolute value.

Results: The majority of patients received clamshell incision $(64.6 \%, n=86)$. Age was similar between the clamshell (38.2 years) and the bilateral anterolateral thoracotomy ( 40.5 years, $p=0.365)$ groups, while the former was characterized by higher LAS ( 39.3 vs $35.1, p=0.035)$. FVC was similar between the two groups before surgery, but it was systematically lower in the clamshell group in the three considered follow-ups (Figure1).

Conclusions: The clamshell incision results in more postoperative lung restriction as compared with bilateral anterolateral thoracotomy; we note that this effect persists throughout the first year.
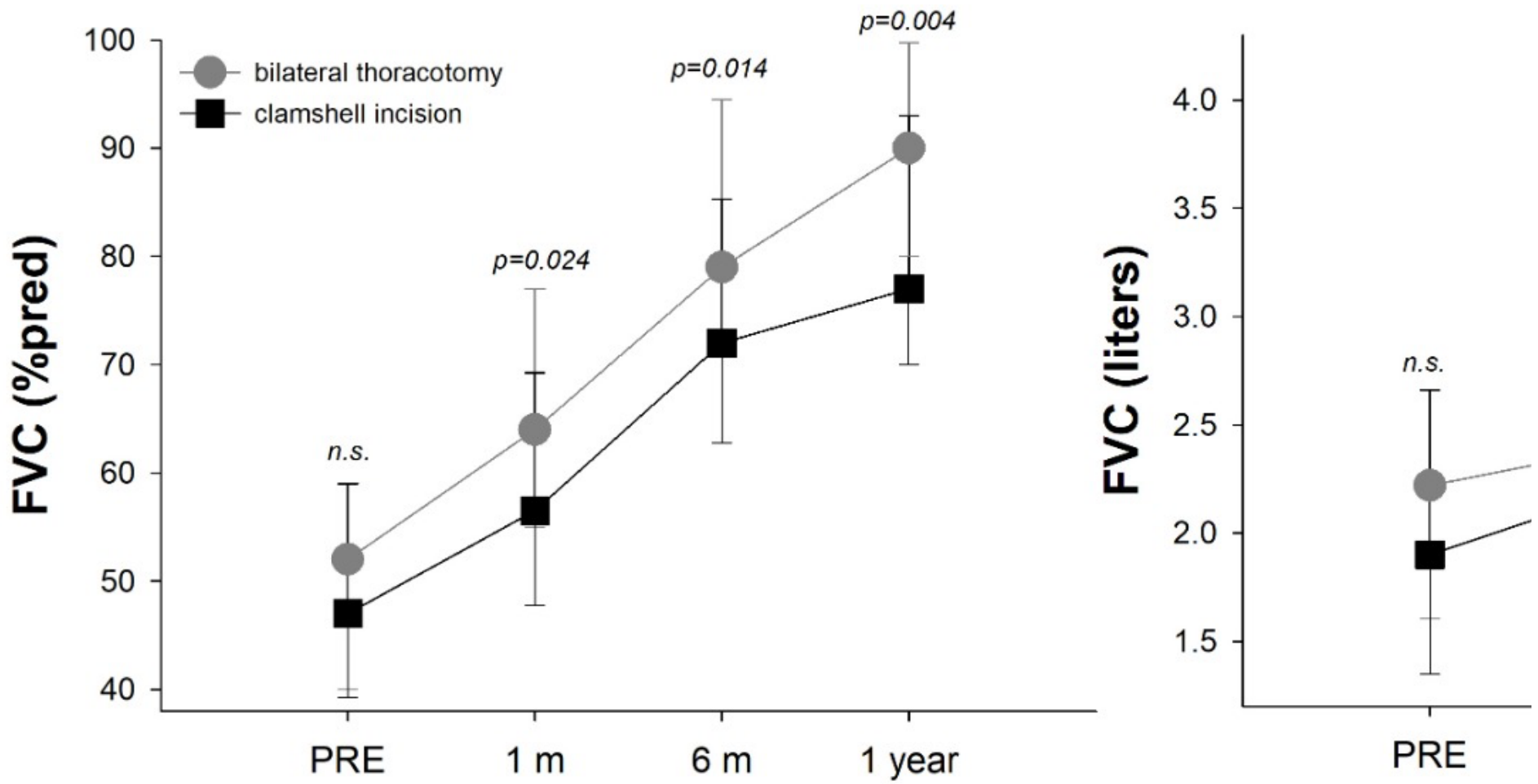



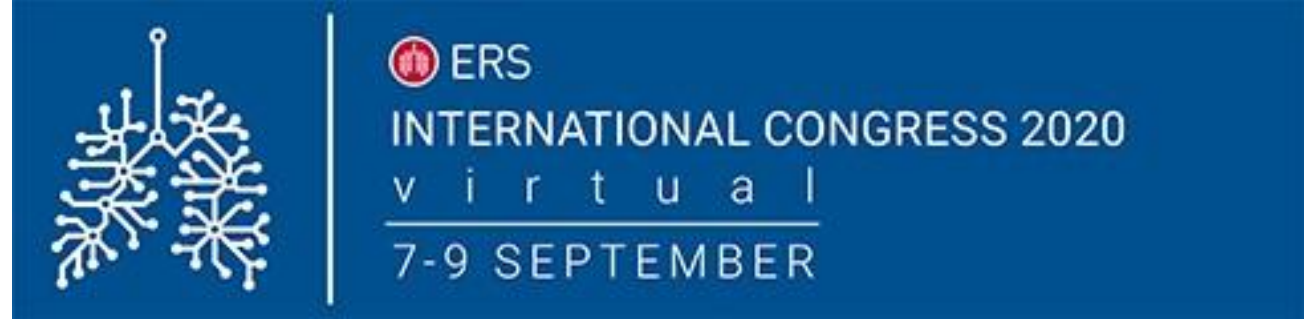

$22 / 07 / 2020$

Dear Dr. Alessandro Palleschi,

We are pleased to inform you that your abstract entitled:

"Clamshell vs bilateral anterolateral thoracotomy for double lung transplantation: impact on vital capacity"

has been selected for presentation in E-poster session at the ERS 2020 Virtual International Congress that will take place from 7-9 September entitled

"Clinical management of lung transplant recipients"

The session will be held on 24/08/2020 from 00:00 to 23:59 in Pre-congress content.

Read carefully the instructions (https://meventscross.freshdesk.com/support/solutions/16000060865) on how to prepare your abstract presentation. Depending in which type of session your abstract is allocated to, you will need to upload specific files prior to the ERS Virtual Congress.

This year following formats of the abstract sessions are included in the congress programme:

- E-poster sessions (former Thematic poster and Poster discussion) - all accepted abstracts will be published on the platform 2 weeks prior to the event in the format of e-Poster with a voice over.

- Oral presentation and ALERT sessions - all accepted abstracts will be published on the platform 2 weeks prior to the event in the format of e-Poster, with a voice over. In addition, the abstracts will be presented in live, moderated oral presentation sessions as PowerPoint presentation. Abstract presenters will attend 1-hour session, where along with session chairs/moderators and the audience will take part in the live, fully virtual, interactive discussion on the presented abstracts. The session chairs/moderators will be kindly asked to comment the e-posters via the congress platform, as well.

We would like to point out that:

1. As an abstract presenter you need to register to the congress to be able to read questions and interact with chairs of the session, as well as with participants. Please go to registration webpage.) ( https://my.ersnet.org/Account/Authentication?Resource=RegisterToCongress\&ReturnUrl=\%2FCongress\%2FMain\%2FDisplayEve nt)

2. No change can be made to the abstract content.

3. Real or perceived conflicts of interest that relate to your presentation have to be disclosed when presenting your abstract (on poster or on slide presentation) and on your E-Poster.

4. If, for any reason, you have to cancel your presentation, it is important that you notify us immediately. Use the following contact details:

- To cancel your abstract presentation both in the Programme and the Publication: ERS Scientific Dept. in Lausanne by e-mail: abstracts@ersnet.org

- To cancel your registration to the Congress contact: by email: ERS Congress Registrations congress.registrations@ersnet.org

- If you will not be the one presenting the abstract, update this information directly on the abstract platform in https://my.ersnet.org/.

Yours sincerely,

ERS Scientific Programme department on behalf of

Daiana Stolz and Christopher E. Brightling

ERS International Congress Programme Committee Chairs 